ISSN 1112-9867

http://www.jfas.info

\title{
A NEW METHOD FOR PROVIDING A MODEL TO ANALYZE A KITE WIND TURBINE
}

\author{
N.Sabet ${ }^{1}$, M. Akbari ${ }^{2}$ and A. Zandvakili, ${ }^{2 *}$ \\ ${ }^{1}$ SAMA Technical and Vocational Training College, Islamic Azad University, Kerman \\ Branch, Kerman, Iran \\ ${ }^{2}$ Faculty of Electrical Engineering, Islamic Azad University, Kerman Branch, Kerman, Iran
}

Published online: 18 June 2016

\begin{abstract}
The ultimate purpose of simulating kite wind turbine is to predict the kite movement and the power generated by its mechanism. To simulate because when the initial estimate of the intended values is found, we may have to manipulate the simulation to find the most efficient design for manufacturing our system and then predict the final power generated for our mechanism. Simulation for the system is based on two parts: Simulation of steady-state and dynamic model, which include differential equations. In this paper, we have presented a novel idea for a proper simulation of the system based on the two static and dynamic parts in order to numerical solution of the required amount and to calculate the output power.
\end{abstract}

Keywords: Kite wind turbine; Simulation; Static model; Dynamic model; Winch; Torque

Author Correspondence, e-mail: nasrin.sbt@gmail.com

doi: http://dx.doi.org/10.4314/jfas.v8i2s.143

\section{INTRODUCTION}

Over the past few decades, the concern about finishing fossil fuels has increased the interest in using renewable energy sources. As a result, the amount of research carried out on various types of renewable sources of energy has grown dramatically. Many researchers believe that wind energy, which is a renewable energy, is a viable alternative for replacing fossil fuels. 
Wind energy despite many advantages has also limitations. Nowadays, the aim is to reduce the costs of this type of energy and to study the feasibility of using kite wind turbines to harness wind energy and converting it into a useful form of mechanical or electrical energy.

Although wind towers have become higher, better and more appropriate, soon their electricity production through a small number of Kite wind turbines will be left behind. Generally, there are two wind flows that surround the Earth completely: One is in the southern half and Tierra del Fuego latitude and the other one passes from Europe and North America. The height of the flows varies from 500 meters up to 10,000 meters - and their width is about 5000- $4000 \mathrm{~km}$. This wind energy is 2 kilowatts per square meter averagely. This means that the wind passing through Europe has a power of 100,000 nuclear power plant. This huge amount of renewable energy took our attention to this matter that how we can capture and use this energy by a series of Kites.

Kite wind turbine is made up of a rotating stimulus large circle which is connected by strong polyethylene cables to earth and is firstly activated by wind. It is noteworthy that light weighted and strong kites can be moved by the chimney of houses or by hand and can be placed at the height of 1000 meters or higher. Central structure of the rotating system includes reels (winch) that automatically release some paired cables to control the kite.

Wind causes sudden and rotational motion of the kite and the generated energy is transmitted to earth by polyethylene cables that is turned into electricity by a generator and injected into the grid [1].

When the kite reached its maximum height, which means the rope placed in a state of maximum tensile, the core of kite by changing the angle of kite and reducing the wind pressure exerted on it causes the stretched cables return to their previous status and prepare for a next cycle to produce energy [2].

Although returning the cables from stretching state back to their normal state requires energy, the energy consumed for this function compared to its energy production is very little.

Thus, as you see in Figure 1, when Paraglider umbrella is exposed to air flow, air enters the holes that are in front of it and then, the umbrella plays the role of a wing, causing it to glide in the air. If the shape of umbrella was like a parachute for example, it was not able to slip in 
the air flow; since parachute due to its structure does not have a flight range and comes down but Paraglider with such structure involves a greater range of motion.

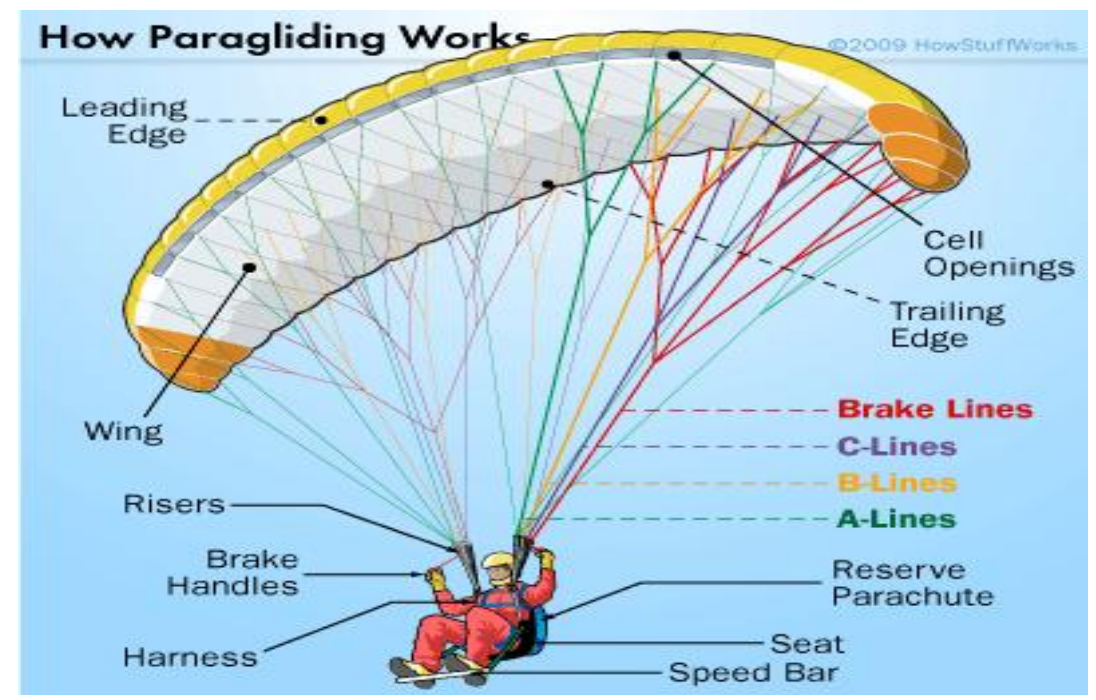

Fig.1. How a Paraglider works

\subsection{Kite testing}

In this setup, first we have connected a Kite to a digital tensometer, which has connected it to the earth, with 1 meter distance. Gaining data quickly from digital tensometer allows us to find rope tension. Since 4-lane Kites have the ability to control the angle of attack, several tensions are measured at different angles of attack. To measure the angle $\theta$, which is used to determine the Kite's approximate angle of attack, a digital protractor is used. However, the problem that makes our experiment almost impossible is measuring the kite's angle of attack in flight [3].

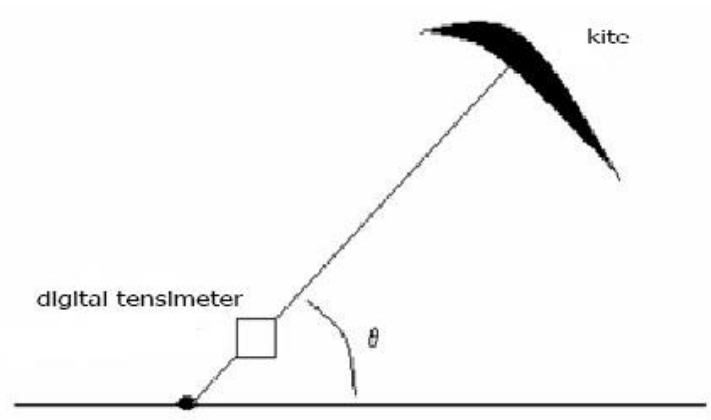

Fig.2. Kite testing

To solve this problem, we assume that the Kite's angle of attack in the non-power mode 
(line's tension $=0$ ) is zero and in the power mode (maximum line's tension) is 90 minus the angle $\theta$ which is measured on the Earth [9].

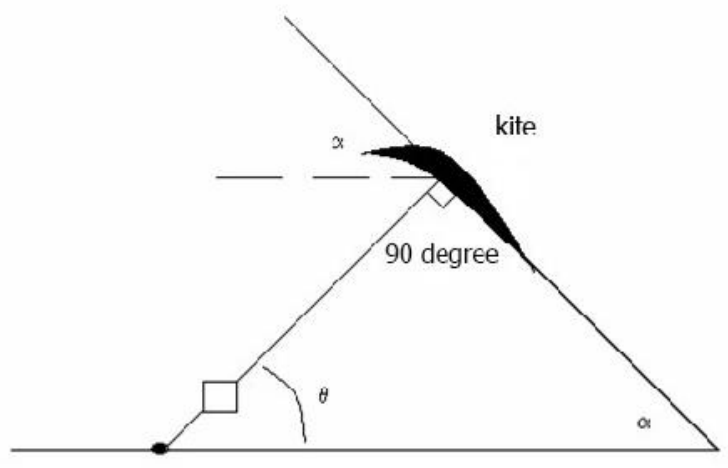

Fig.3. Approximate angle of attack

Since the angle of flow in a Kite is obtained according to the following equation:

$$
\frac{x}{y}=\sin \alpha
$$

$\mathrm{X}$ is longitudinal distance of both sides of the Kite, $\mathrm{y}$ chord length or the Kite's height to the fixed part, $\alpha$ the kite's angle of flow and $\theta$ is the Kite's approximate angle of attack:

$$
\gamma=11 \cdot-4 \cdot-\theta
$$

This approximation is not always accurate because the kite can be already at one angle and this is possible only in non-power mode (tension is 0). However, for this purpose, this approximation is accurate enough to determine an overall shape of the kite, in contrast with relative angle of attack [4].

\subsection{Steady-state simulation}

We perform the Kite modeling with the steady-state based on the work we have already explained. Some of the important variables are defined in Figure 4:

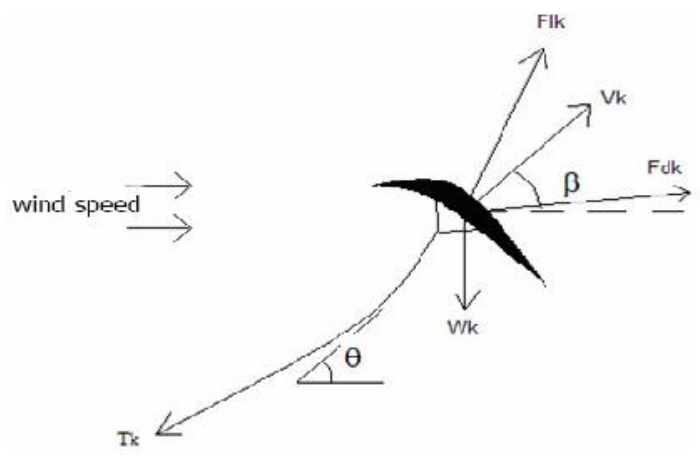

Fig.4. The Kite model 
It is noteworthy that the analysis of the steady-state dependents on its oscillation mode [5]. The variables used in the calculations of the kite's steady-state are as follows:

Where $\mathrm{V}$ is wind speed, Flk is the kite lift force, Wk the kite weight, $\mathrm{g}$ the gravitational constant, $\beta$ the kite's angle of motion relative to the earth, $\theta$ the kite's angle relative to the earth, AK the kite's level, $\alpha$ the kite's hypothetical angle of attack, AR the kite's dimensions ratio, eo the assumed value for tension calculations, Cdo hypothetical drag coefficients.

The purpose of steady-state calculations is to find the rope tension created by the kite that the kite's normalized speed, the kite's normalized weight and increased drag ratio need to develop an equation for the kite's rope tension [6].

At first, of the kite normalized speed determines the kite's speed. According to the equation:

$$
V_{w r}=\sqrt{V=V k-2 V V k \cdot \cos (\beta)}
$$

To calculate the kite's speed according to the arm's motion, the following equation is used:

$$
V k=\frac{R_{a} \cdot \omega}{\cos \left(\gamma-\beta+\frac{\pi}{2}\right)}
$$

Where $\mathrm{Ra}$ is the length of pivot point up to the kite tether, $\omega$ angular velocity of the arm rotation, $\gamma$ the angle between the arm and the surface of Earth. With the total speed of the kite, we can find a value for the kite normal speed.

$$
V_{w r n o r m}=\frac{V_{w r}}{V}
$$

To increase the drag coefficients, modeling equations of airfoils' aerodynamic performance was added to analyze the kite's steady-state. A wide range of angles of attack were simulated using the ratio of the kite upward tension, which ultimately we could simulate it at different angles of attack.

This range of values $\mathrm{L} / \mathrm{D}$ can be determined using the basic theory of airfoil and limited wing theory. By this theory we can also approximate the values of CI and Cd which determine L/D. Coefficient of lifting (CL) using the basic theory of airfoil:

$$
C l=\left(\frac{d}{d \alpha} C l\right) \cdot\left(\alpha-\alpha_{\text {zeroLift }}\right)
$$

Using the derived wing theory, lifting coefficients in the equation are assumed as follows: 


$$
\frac{d}{d \alpha} C l=\frac{2 \cdot \pi}{1+\frac{2 \cdot \pi}{\pi \cdot A R}}
$$

By substituting equation (6) in equation (7) lifting coefficients (CI) are obtained:

$$
C l=\frac{2 \cdot \pi \cdot \alpha}{1+\frac{2}{A R}}
$$

Drag coefficient $(\mathrm{Cd})$ also by following equation:

$$
C d=C d o+\frac{c l^{2}}{\pi \cdot A R \cdot e o}
$$

Using the calculated values of $\mathrm{Cd}$ and $\mathrm{CD}$, we can find a wide range of the rope tension ratio:

$\frac{L}{D}=\frac{C l}{C d}$

By reversing the above equation:

$$
\frac{D}{L}=\frac{1}{\frac{L}{D}}
$$

Equations (1) and (11) show upward and downward drag coefficients that follows the kite angle of attack $(\alpha)$. The last step is to find the values of the kite normal weight through the following equation:

$$
W_{k n o r m}=\frac{W k \cdot g}{0.5 \cdot \rho V \cdot C l \cdot A k}
$$

Finally, the normal drag coefficient $T_{L}$ of rope is obtained:

$$
T L=\sqrt{\text { wrnorm }{ }^{4}\left[\left(\frac{D}{L}\right)+1\right]+\text { W knorm }^{2}-2 W \text { knorm Vwrnorm }\left[1-V_{\&} \cdot \cos (\beta)-\left(\frac{D}{L}\right) V, \cdot \sin (\beta)\right]}
$$

And by using this equation we can find the numerical value of tension:

$$
T=T L \cdot 0.5 \cdot \rho \cdot V^{2} \cdot C l \cdot A k
$$

Forces acting on the kite can be estimated using the rope tension from the kite steady-state model. Additional parameters are used to develop oscillation model of the rod.

The kite steady-state equations to simulate its static part are based on a model that can be seen in Figure 5: 


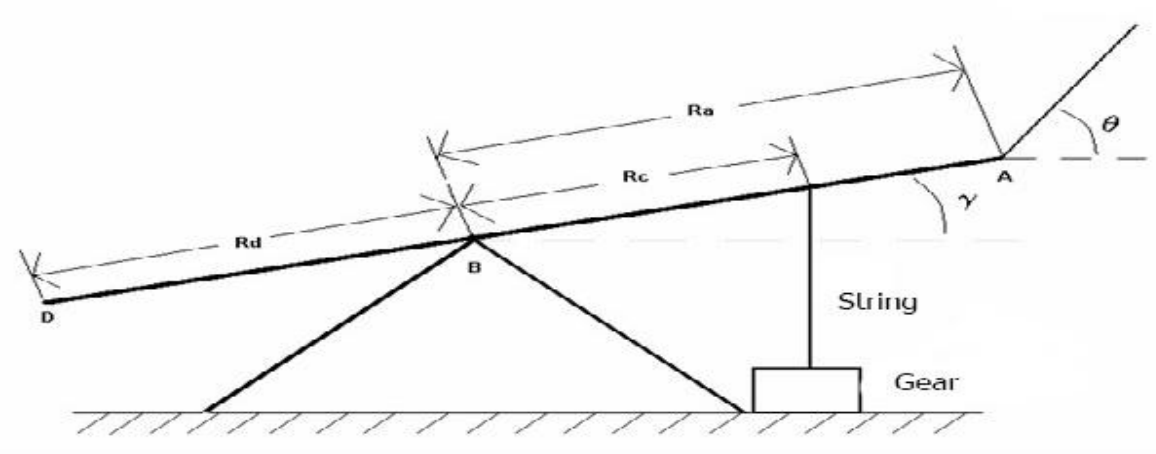

Fig.5. Diagram of the pivot arm

Values which are used to simulate the pivot arm:

Where $\mathrm{Ra}$ is the Length point $\mathrm{A}$ up to point $\mathrm{B}, \theta$ the kite angle against the earth, $\mathrm{RC}$ the arm length of point $\mathrm{B}$ up to string, $\beta$ the kite motion angle to the earth, $\mathrm{W}-\mathrm{DA}$ the Arm weight between $\mathrm{D}$ and $\mathrm{A}, \omega$ angular speed of the arm rotation, $\mathrm{W}-\mathrm{BA}$ the Arm weight between $\mathrm{A}$ and $\mathrm{B}, \gamma$ the angle between the arm and surface of the earth, $W^{\gamma}$ Weight of the angle of attack, TK the string drag from the kite side and TC is the string drag. Total of pivot point of the rotating arm by calculating the tension created in the string attached to the gear system is possible through calculating the line drag from the steady-state theory, which is as follows:

$$
T_{c} \cdot \cos \left(\gamma-\theta+\frac{\pi}{2}\right) \cdot R a-T_{c} \cdot \cos (\gamma) \cdot R_{c}+W{ }_{-} D B \cdot \cos (\gamma) \cdot \frac{R_{d}}{2}-W{ }_{-} B A \cdot \cos (\gamma) \cdot \frac{R_{a}}{2}-W 2 \cdot \cos (\gamma) \cdot R 2=0
$$

By solving the function of string tensions we have:

$$
T_{c}=\frac{T 2 \cdot \cos \left(\gamma-\theta+\frac{\pi}{2}\right) \cdot R a+\left(g W-D B \cdot \cos (\gamma) \cdot \frac{R_{d}}{2}-g W \quad B A \cdot \cos (\gamma) \cdot \frac{R_{a}}{2}-W 2 \cdot \cos (\gamma) \cdot R 2 \cdot g\right)}{\cos (\gamma) \cdot R_{c}}=0
$$

The rope drag can be determined using the above equation.

The next step is to use the rope drags, which are caused by the motion of the kite under the influence of flow, to estimate the torque and rotary speed of a generator shaft. A simple gear system has been shown in figure (6):

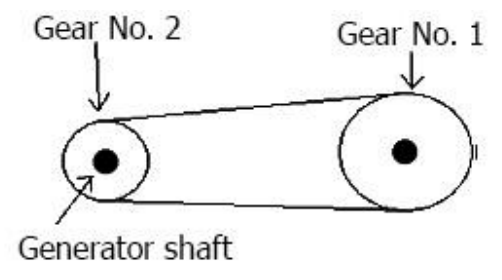

Fig.6. Dual-gear system Schematics 
In above gear system, the rope attached to the rod rotates the shaft on the gear wheel $\nexists$, and gives the amount of torque and RPM to the gear $\neq 2$. The following values are defined for this gear system:

Where $S t$ is movement time, $Q^{\prime}$ number of teeth on the first shaft, $S I$ length of movement, $Q^{\top}$ number of teeth on the second shaft, $r^{\prime}$ radius of the first gear, $r^{\top}$ radius of the second gear, $T^{\varepsilon}$ Torque of generator shaft, $R P M^{\uparrow}$ rotary speed of the first shaft, $R P M^{\uparrow}$ is rotary speed of the second shaft. The torque on the gear wheel is typically approximated by the product of the rope drag and radius of the gear. From this approximation we can obtain the torque on the shaft of the generator using the following equation:

$$
T 4=T c \cdot r 2
$$

Predicting the generator shaft time depends on the movement time of the arm rotation, so that movement through power phase is approximated by finding the time required for kite. Gear times $\nexists$ and gear times $\not 2$ is obtained through the following equation:

$R P M 1=\frac{\frac{S l .60}{S t}}{2 . \pi \cdot r 1}$

With the amount of gear times $1 \neq$, the time created on the gear $2 \neq$ can be estimated.

$$
R P M 2=\left(\frac{Q 1}{Q 3}\right) \cdot R P M 1
$$

This is final step in the steady-state theory. Results of the Kite steady-state theory model and dynamic model will be given in the next section.

\subsection{Dynamic model simulation}

Dynamics simulation of our system takes a deeper look at the power generation and overall performance of all the mechanism. The main advantage of this system compared to steady-state theory is that it allows us to predict performance of the system. For dynamic modeling, we made use of a system that included a load at the end of a rod which is an alternative for the Kite tension [7,8,10]. Modified mechanism diagram has been shown in 
Figure 7:

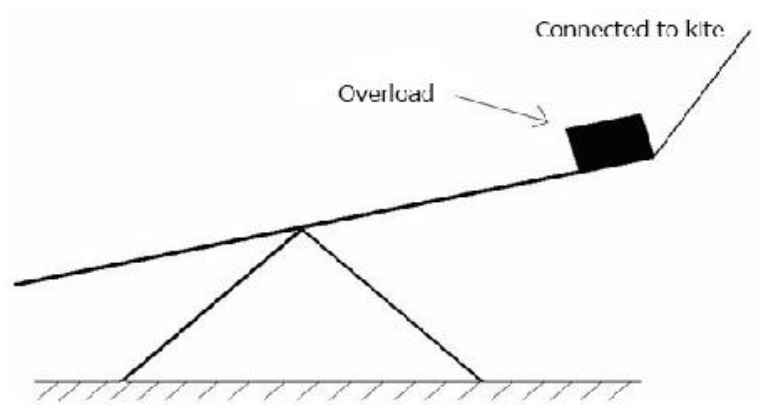

Fig.7. The modified system simulation

Here the overall system power will be based on the mechanical power generated by the rotating arm. This power can be approximated using the following equation:

$$
P=T . \omega
$$

Where $\mathrm{T}$ is torque of the rotating rod and $\omega$ is rotary speed of the rotating rod.

Mathematical equations used to simulate the Kite dynamic section are calculated by the Kite start moving. Using Figure 8, two coordinates have been defined:

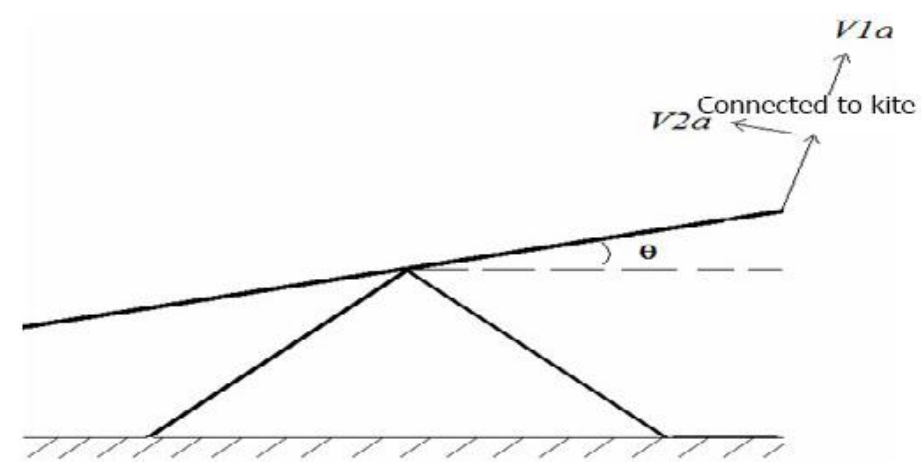

Fig.8. Simulation of parameters

In this figure, $V^{\prime} a$ represents the kite speed toward the rope attached to the kite. While $V^{\top} a$ represents the kite perpendicular to the rope attached to the kite. By using equation (21) to change the speed and direction of $V^{\top} a$, we find that:

$$
\frac{d}{d t} V 2 a=\left(\frac{g}{w k}\right)\left(F_{-} d k \cdot \sin (\theta+\phi)\right)-F_{-} l k \cdot \cos (\theta+\phi)+W k \cdot \cos (\theta)+\frac{2 W k V 1 a V 2 a}{g \cdot L 1}
$$

Since the kite is attached to the rod, it is clear that no speed or movement exist toward $V^{\prime} a$ direction. Thus, the rotational force can be ignored due to the speed in the direction of $V^{\prime} a$ : 


$$
V 1 a=0 \quad \frac{d}{d t} V 1 a=0
$$

Therefore, changing the kite speed in the direction of $V^{r} a$ is as follows:

$$
\frac{d}{d t} V 2 a=\left(\frac{g}{w k}\right)\left(F_{-} d k \cdot \sin (\theta+\phi)-F_{-} l k \cdot \cos (\theta+\phi)+W k \cdot \cos (\theta)\right)
$$

According to $V^{i} a$ direction which has been shown in (Goela et al., 1986) theory, changing the kite speed is assumed as follows:

$$
\frac{d}{d t} V 1 a=\left(\frac{g}{w k}\right)\left(F_{-} d k \cdot \sin (\theta+\phi)-F_{-} l k \cdot \cos (\theta+\phi)+W k \cdot \cos (\theta)+\frac{2 W k V 1 a V 2 a}{g \cdot L 1}\right)+\left(W k \cdot \frac{V 2 a^{2}}{g \cdot L 1}\right)-F t
$$

Since the derivative of $V^{\prime} a$ is a value equal to zero, this equation can be re-arranged to calculate the kite tension that the kite tension is obtained through the following equation:

$$
F t=F d k \cdot \cos (\theta+\phi)+F l k \sin (\theta+\phi) W k \sin (\theta)+W k \cdot \frac{V 2 a^{2}}{g \cdot L 1}-F t
$$

Using equations that we have already mentioned, the kite dynamic mode can be easily simulated. The next step is to attach the kite dynamics to oscillating motion of the arm. A change in the angle $\theta$ is calculated based on the kite movement speed.

$$
-V \quad 2 a=\dot{\theta} \cdot L 1
$$

Which to express generally the following form is arranged;

$$
\frac{d}{d t} \theta=\frac{-V 2 a}{L 1}
$$

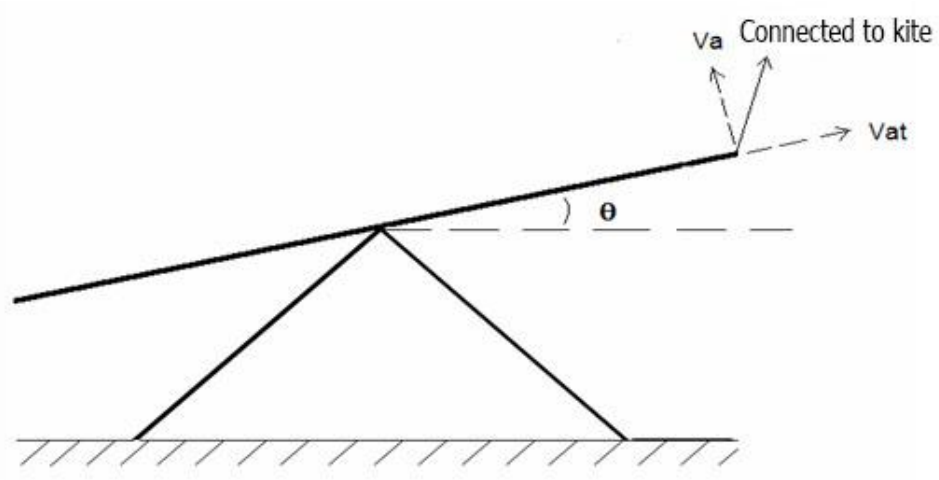

Fig.9. Simulated parameters of Part \# 2 
In this figure, $\mathrm{V}_{\mathrm{a}}$ represents the speed of rod perpendicular on it and Vat represents the speed of rod parallel to the rod direction. In this case, sum of the rod rotation's moments reaches a peak, which is shown in the following equation:

$$
\frac{-1}{g} \cdot\left(W d a+I_{-} A D \cdot \frac{g}{R A}\right) \cdot\left(\frac{d}{d t} V a\right)-\left(\frac{2}{g}\right) \cdot\left(W \_D A+I_{-} A D \cdot \frac{g}{L L^{2}}\right) \operatorname{Vat} \cdot \frac{V a}{L L}=F \cdot \cos \left(\gamma-\theta+\frac{\pi}{2}\right) R A-F c \cdot \cos (\gamma) \cdot R C
$$

In this case, it is clear that the rod does not have any movement in Vat since the rod is in a fixed rotary form. Therefore, it can be assumed that the speed toward $V_{a}$ is obtained through the following equation:

$$
V a:=R A . \omega
$$

Angular velocity of the rod can be calculated using following equations:

$$
\begin{gathered}
\omega:=\frac{d}{d t} \gamma \\
\frac{d}{d t} \gamma:=\frac{V a}{R A}
\end{gathered}
$$

Mechanical power of system can be calculated based on the rotation of the rod.

$$
P=T \omega
$$

By substituting angular velocity, power can be obtained:

$$
P=T \frac{V a}{R A}
$$

The main key of simulation is based on the four differential equations (25), (28), (29) and (30). Using these four equations and setting some initial values, we can calculate and measure the Kite tension and dynamics of oscillating rod.

For simulation we can use MATLAB and/or FLUENT software. This means that by using MATLAB, a time step is passed to determine the number of repetitions in differential equations. When the initial values are determined, MATLAB simulates the equations with more than a time limit for the dynamic system.

One of the difficulties with these equations is that they are not included the angle of attack, which means that they only describe the rod and the kite movement just in one of their directions either up or down. Thus, MATLAB is adjusted for a point where oscillating rod 
reaches a certain height and then the Kite angle of attack changes. Once the rod made an angle by the horizontal surface, it changes the code of the Kite angle of attack to respond to changing of the rod angle with surface. For example, when rotation in accordance with mathematical rules reaches a downward angle toward the horizon, the Kite angle of attack changes to a higher value and launches the Kite to provide power that is shown in the following figure as an example:

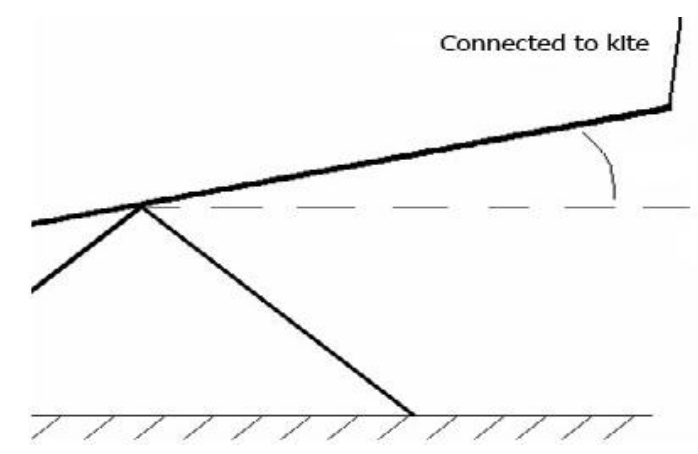

Fig.10. Rotating rod that reaches to the specified angle in the program

Fluent software is also capable of performing calculations with both normal precision and double precision and user can select each as an option. In addition to greater speed of numerical calculations, full and more detailed information, such as changes in velocity, pressure, temperature, etc. across the desired field can be easily achieved through these methods.

\section{CONCLUSION}

Since the simulation idea for the Kite wind turbine system is based on static and dynamic models, it includes various differential equations. To simulate this system and energy production through it, creating a computer model and its numerical analysis in order to achieve a result is desirable. For this purpose, by using its analysis in a more simple form and considering the system as being constant, we can easily perform numerical analysis of the system and then, for overall simulation of the system in the varying mode, we can use MATLAB and FLUENT software to analyze and modeling the kite and to calculate the drag force resulting from the rope attached to it. 


\section{ACKNOWLEDGEMENTS}

The authors would thank Professor Farshid Keynia and Hossein Amiri for their insightful reading and constructive suggestions.

\section{REFERENCES}

[1] Boyer, M. Altaeros Energies' Floating Wind Turbines. Tap Into Strong High Altitude Winds",Inhabitat, 28 March 2012, Retrieved , 9 July 2012.

[2] Manalis, M.S. 1975. Airborne Windmills: Energy Source for Communication Aerostats. AIAA Lighter Than Air Technology Conference, AIAA Paper, 1975

[3] Lang, David, Electrical Power Generation Using Kites. Drachen Foundation, 2005.

[4] Varma, S.K., and Goela, J.S. Effect of Wind Loading on the Design of a Kite Tether. Journal of Energy, 1982, 4, 113.

[5] Canale.M, Fagiano.L, Milanese.M, /high Altitude Wind Energy Generation Using Controlled Power Kites, IEEE Transactions on control systems Technology, 2008.

[6] Pugh, P.F.Wind Generator Kite System, US Patent 4, 1984.

[7] Kumar, R.V.Wind Loading Effects on a Catenary, Journal of Wind Engineering and Industrial Aerodynamics, 1985, 22, 234.

[8] Hoisington, Z.C.Variable Surface Area Paraglider, 1999, AIAA Paper 99-0009.

[9] Lansdorp, B., and Ockels, W.J.Comparison of Concepts for High-Altitude Wind Energy Generation with Ground Based Generator, Proceedings of the NRE, Conference Beijing, China , 2005, 409-417.

[10] Slice of MIT,"Two Alums Dream of Tethered Aerostat Wind", two alums dreaming of tethered aerostat wind farms/ Retrieved, 2013.

\section{How to cite this article:}

Sabet N, Akbari M, Zandvakili A. A New Method For Providing A Model To Analyze A Kite Wind Turbine. J. Fundam. Appl. Sci., 2016, 8(2S), 901-913. 